\title{
Entre tournant réaliste et nouveau radicalisme : les syndicats, le Parti travailliste et l'État britannique, de la révolution thatchérienne au néo-travaillisme
}

Between the return to realism and new radicalism: unions, Labour and the British State, from the thatcherite revolution to New Labour

\section{Marc Lenormand}

\section{OpenEdition Journals}

Édition électronique

URL : http://journals.openedition.org/rfcb/1148

DOI : $10.4000 /$ rfcb. 1148

ISSN : 2429-4373

Éditeur

CRECIB - Centre de recherche et d'études en civilisation britannique

\section{Édition imprimée}

Date de publication : 27 juillet 2009

Pagination : 167-187

ISBN : 978-2-9115-8028-4

ISSN : 0248-9015

Référence électronique

Marc Lenormand, « Entre tournant réaliste et nouveau radicalisme : les syndicats, le Parti travailliste et l'État britannique, de la révolution thatchérienne au néo-travaillisme », Revue Française de Civilisation Britannique [En ligne], XV-2 | 2009, mis en ligne le 01 novembre 2016, consulté le 19 avril 2019. URL http://journals.openedition.org/rfcb/1148; DOI : 10.4000/rfcb.1148

\section{(c) $\mathbb{( \Theta \Theta}$}

Revue française de civilisation britannique est mis à disposition selon les termes de la licence Creative Commons Attribution - Pas d'Utilisation Commerciale - Pas de Modification 4.0 International. 


\title{
Entre tournant réaliste et nouveau radicalisme : les syndicats, le Parti travailliste et l'État britannique, de la révolution thatchérienne au néo-travaillisme
}

\author{
Marc LENORMAND \\ Université Paris 8
}

\section{Trente ans après}

La Grande-Bretagne est paralysée par un mouvement de grève qui touche près de 600000 salariés du secteur public. Bibliothécaires, assistantes sociales, ramasseurs d'ordures, cantonniers, agents de nettoyage, cuisiniers et géomètres protestent contre des augmentations de salaires inférieures à l'inflation. Un gouvernement travailliste malmené dans les sondages fait face au mécontentement des syndicats, avec lesquels les relations se sont détériorées du fait de l'application stricte de politiques de rigueur budgétaire au secteur public ${ }^{1}$. La désaffection vis-àvis du gouvernement travailliste s'étend dans les rangs de son électorat ouvrier traditionnel. Les tensions sont telles que des centrales syndicales parmi les plus puissantes envisagent de réduire, voire mettre un terme au soutien financier qu'elles apportent au Parti travailliste ${ }^{2}$. En effet, comme tous les quotidiens se plaisent à le souligner, les syndicats sont les trésoriers du Parti travailliste, et donc son véritable maitre ${ }^{3}$. En retour de leur soutien financier et organisationnel au parti, ils exigent du gouvernement travailliste non seulement qu'il cède à leurs revendications salariales, mais qu'il mette en œuvre des politiques sociales et fiscales plus redistributrices, et

\footnotetext{
${ }^{1}$ Jonathan OLIVER, 'Trade union chiefs ready to dump Brown', The Times, 20 juillet 2008 ; Sam COATES, 'Unions call for Business Secretary John Hutton's head after "fall-out"', The Times, 23 juillet 2008 ; David HENCKE, 'Talks on future of public services turns fractious', Guardian, 26 juillet 2008.

2 'Public sector workers join exodus from Labour', Daily Telegraph, 19 juin 2008 ; Christine BUCKLEY, 'Big unions being urged by activists to split from the party', The Times, 19 mai 2008 ; 'Union “dismayed” at PM's leadership', Daily Express, 29 mai 2008.

${ }^{3}$ David HENCKE, 'Political party funding : union cuts funds to MPs it says do not support Labour values', Guardian, 10 juin 2008 ; Alan JONES, 'GMB threatens Labour MPs' funds', The Independent, 10 juin 2008 ; James KIRKUP, 'Labour "too dependent on trade unions"', Daily Telegraph, 30 juin 2008 ; The Guardian, 'Power follows money', Guardian, 3 juillet 2008 ; Rosa PRINCE, 'Unison union leaders threaten to withdraw Labour funding', Daily Telegraph, 10 juillet 2008 ; Andrew PIERCE \& Richard TYLER, 'Trade unions cash support for Labour in return for policy changes', Daily Telegraph, 13 juillet 2008.
} 
qu'il soutienne le développement de l'activité syndicale dans les entreprises ${ }^{4}$. Alors que le gouvernement, arc-bouté sur sa politique d'austérité budgétaire, refuse de répondre favorablement aux exigences des syndicats, la presse annonce des jours sombres pour l'économie et les citoyens britanniques, prisonniers de l'intransigeance du gouvernement et des tactiques agressives des syndicats ${ }^{5}$.

On pourrait croire qu'il s'agit d'un aperçu des images véhiculées par les médias pendant l'« hiver du mécontentement» de 1978-1979. Il s'agit en fait des analyses proposées par la presse lors de conflits dans le secteur public pendant l'été 2008. Trente ans séparent ces deux mouvements de grogne, et pourtant le vocabulaire et les logiques argumentatives des quotidiens britanniques sont demeurées les mêmes. Après avoir annoncé successivement un «automne du mécontentement » et un «nouvel hiver du mécontentement » fin 2007, le Daily Mail titre en avril 2008: «Voici venu l'été du mécontentement», pendant que le Daily Telegraph s'interroge : « 2008 sera-t-il l'été du mécontentement ?» ${ }^{6}$. À en croire la presse, les revendications des salariés sont les mêmes qu'en 1978-1979, le rapport de force entre les syndicats et le gouvernement aussi. Faut-il alors en déduire que les relations entre les syndicats et un gouvernement travailliste n'ont pas été affectées par dix-huit années de révolution thatchérienne dans la gestion des rapports sociaux, et dix années de consolidation et d'adaptation blairiste de ce nouvel ordre sociopolitique?

À y regarder de plus près, les deux conflits sont pourtant différents. En dépit de l'inflation de la couverture médiatique, le mouvement de l'été 2008 a consisté en un arrêt de travail de deux jours seulement, bien loin des grèves roulantes qui se sont poursuivies pendant plusieurs semaines entre fin janvier et début mars 1979. À côté des questions salariales, les revendications exprimées par les syndicats révèlent un rapport de forces très différent au sein du monde du travail. À la fin des années 1970, les organisations de travailleurs souhaitent en effet aller plus loin dans la démocratisation des rapports sociaux. S'il n'y a pas consensus au sein du mouvement syndical autour de la nécessité de la démocratie industrielle (industrial democracy) et des modalités que celle-ci doit prendre, les syndicats s'accordent sur le fait que les garanties offertes en matière de droits syndicaux par la législation travailliste de 1974-1976 sont insuffisantes, et que le gouvernement doit poursuivre la mise en place de dispositifs encourageant la syndicalisation. Au cours de l'été 2008, en revanche, les syndicats demandent seulement du gouvernement travailliste qu'il les libère des aspects les plus contraignants et les plus punitifs de la législation anti-syndicale mise en œuvre par les gouvernements conservateurs entre 1980 et

\footnotetext{
${ }^{4}$ Patrick WINTOUR \& Nicholas WATT, 'Unions put new demands to Brown', Guardian, 30 juin 2008 ; 'If you earn $£ 40,000$ you can afford to pay higher taxes, say unions', Daily Mail, 1 juillet 2008 ; Ryan KISIEL, 'Ministers bow to union leaders over new rights for workers', Daily Mail, 15 juillet 2008 ; Andrew GRICE, Ben RUSSELL \& Andy MCSMITH, 'As strike bites, unions press Brown to raise taxes on rich', Independent, 17 juillet 2008.

${ }^{5}$ George PASCOE-WATSON, 'Brown snubs union strike call', Sun, 7 juillet 2008 ; Andrew GRICE, 'Brown to reject calls for tax rise for higher paid', Independent, 25 juillet 2008.

${ }^{6}$ Laura CLARK \& Ian DRURY, 'Here comes the summer of our discontent: As teachers' strike cripples our schools, Brown is warned of further public sector walkouts', Daily Mail, 25 avril 2008 ; 'Will 2008 be a summer of discontent?', Daily Telegraph, 23 juin 2008.
} 
$1993^{7}$. Le point de départ n'est plus le même : en 1978, les syndicats, forts de la progression et de l'élargissement quasi-constants de leurs capacités d'action depuis le début du siècle, veulent obtenir pour les travailleurs une participation plus grande aux décisions qui affectent leurs lieux de travail ; en 2008, au terme de trente année de démantèlement des institutions de régulation collective des rapports sociaux, les syndicats souhaitent se voir restituées quelques-unes des armes dont ils ont été dépossédés depuis les années 1980 et qui permettraient aux salariés de mener une lutte moins inégale face à leurs employeurs.

Cet article propose, en partant de l'analyse d'un contexte social, économique et politique en profonde mutation depuis les années 1980, d'interroger l'évolution de l'attitude des syndicats britanniques face aux institutions étatiques, aux pratiques gouvernementales et aux partis politiques. Dans un contexte d'ébranlement du système socio-politique prévalant depuis l'après-guerre et de démantèlement des institutions pluralistes de gestion des rapports sociaux, et face à l'affaiblissement numérique, économique et politique qui en résulte pour l'organisation collective des travailleurs, un premier champ d'investigation concerne les stratégies d'adaptation du mouvement syndical britannique. Un deuxième axe de recherche concerne l'inflexion et la renégociation du rapport entretenu par les syndicats aux institutions étatiques. Un troisième objet d'études est la reconfiguration de la relation entre les syndicats et le Parti travailliste, c'est-à-dire comment le lien ombilical qui les unit, aussi ancien et fort que controversé et souvent acrimonieux, a été affecté par les programmes successifs de modernisation du parti, notamment par le projet néotravailliste de reconquête du pouvoir.

\section{Le projet thatchérien et l'affaiblissement du mouvement syndical}

En matière de rapports sociaux, le programme des gouvernements conservateurs au pouvoir entre 1979 et 1997 consiste en une remise en cause radicale du système de régulation collective des rapports sociaux progressivement mis en place par les gouvernements de tous bords, depuis la fin du XIX ${ }^{\mathrm{e}}$ siècle. Il est donc nécessaire d'indiquer ici brièvement en quoi consistaient ces institutions et pratiques qui organisaient les rapports entre employeurs et travailleurs avant la révolution thatchérienne. À partir de la fin du XIX ${ }^{\mathrm{e}}$ siècle et des premières difficultés éprouvées par les secteurs manufacturiers traditionnels - filature et textile principalement - un système de régulation collective des rapports sociaux se met en place. Le gouvernement britannique, notamment par l'entremise du Board of Trade (ministère du commerce), encourage les organisations de travailleurs et les employeurs à se réunir pour négocier des salaires et des conditions de travail applicables à l'ensemble de l'industrie, afin d'éviter la concurrence exacerbée entre une multitude de petites entreprises familiales et la conflictualité sociale qui en résulte $^{8}$. Ces négociations annuelles, qui s'étendent progressivement à tous les secteurs de l'économie et rassemblent les directions syndicales et patronales et

${ }^{7}$ James KIRKUP 'Trade unions demand strike action rights in return for Labour Party donations', Daily Telegraph, 3 juillet 2008 ; Patrick WINTOUR, 'Unions hit Brown with 130 demands', Guardian, 18 juillet.

${ }^{8}$ Chris HOWELL, Trade Unions and the State: The Construction of Industrial Relations Institutions in Britain, 1890-2000. Princeton: Princeton University Press, 2005, pp. 56-58. 
souvent des représentants du gouvernement, ponctuent le calendrier social et politique en Grande-Bretagne jusqu'aux années 1980. Cet échelon national de régulation collective se complexifie suite au développement, dans l'entre-deuxguerres et plus encore à partir des années 1950, d'un nouveau modèle industriel basé sur de grandes entreprises fordistes, pour lesquelles la priorité n'est plus de se prémunir de la baisse des coûts salariaux chez leurs concurrents immédiats, mais de pouvoir constamment ajuster la chaîne de production à l'évolution du marché et d'améliorer la productivité. Il leur est donc nécessaire de négocier directement dans les ateliers avec les travailleurs, par l'intermédiaire de leurs délégués (stewards) ${ }^{9}$. Ce système de représentation directe des travailleurs se met en place dès l'entre-deuxguerres dans l'industrie automobile, pour s'étendre dans les années 1950 et 1960 à l'ensemble du secteur manufacturier, avant de s'imposer finalement jusque dans le secteur public dans les années 1970. Dans les secteurs où la syndicalisation est la plus forte, les rapports sociaux sont donc basés sur ce système de régulation collective largement décentralisé, où les salaires et conditions de travail sont déterminés en fonction du rapport de forces établi par les organisations de travailleurs. Dans les secteurs où l'organisation des travailleurs est moins solide, le transport routier et le secteur public par exemple, des instances nationales - les wages councils - déterminent des conditions de travail uniformes pour le secteur ${ }^{10}$. Les wages councils sont cependant pensés comme des exceptions à une vision générale des rapports sociaux fondée non pas sur des droits positifs et individuels, mais sur la capacité des syndicats à établir un rapport de forces favorable aux travailleurs. Pour ce faire, ils bénéficient depuis 1906 d'immunités dans le cadre de conflits sociaux, qui les protègent d'une common law qui tend à ne reconnaitre que les contrats passés entre des individus, et à regarder l'action collective des travailleurs comme une activité criminelle ${ }^{11}$.

À partir de leur arrivée au pouvoir en 1979, les conservateurs entreprennent de démanteler progressivement ce système de régulation collective des rapports sociaux. Entre 1980 et 1993, pas moins de huit lois se succèdent, qui chacune affaiblit un peu plus les capacités d'action des syndicats et multiplie les entraves à l'organisation collective des travailleurs. L'Employment Act de 1980 réduit l'éventail des tactiques légales utilisables par les syndicats dans le cadre d'un conflit social, et encadre sévèrement le monopole d'embauche obtenu par les syndicats dans un nombre croissant d'entreprises dans les années 1980. En 1982, un deuxième Employment Act met fin aux immunités dont les syndicats bénéficiaient depuis 1906 dans le cadre d'un conflit opposant des travailleurs à leur employeur ${ }^{12}$. C'est probablement la pièce maîtresse de l'arsenal anti-syndical mis en place par les conservateurs, dans la mesure où il rend les syndicats vulnérables à des poursuites

\footnotetext{
${ }^{9}$ Ibid., p. 87.

${ }^{10}$ Ibid., p. 92. Les wages councils, prévus par le Wages Councils Act de 1945, remplacent les trade boards créés au sortir de la Première guerre mondiale, et démantelés pour nombre d'entre eux pendant la dépression des années 1930. Les wages councils sont institués dans les secteurs où, du fait de la faiblesse des organisations syndicales et patronales, des mécanismes de libre négociation ne peuvent être mis en place.

${ }^{11}$ Ibid., pp. 61-62.

${ }^{12}$ Richard TAYLOR, The Trade Union Question in British Politics, Oxford : Blackwell, 1993, pp. 321-322.
} 
devant les tribunaux. Des syndicats parmi les plus puissants et les plus militants, tel le syndicat des ouvriers de l'impression, sont ainsi mis au pas au milieu des années 1980 par la saisie de leurs fonds. En 1984, un Trade Union Act remet en cause les principes de démocratie directe des syndicats, en imposant un vote à bulletin secret pour les élections des directions syndicales, la décision de s'engager dans un conflit social, et la constitution d'un fonds politique destiné à financer un parti politique ${ }^{13}$. Entre 1986 et 1993, cinq nouvelles lois se succèdent, qui finissent de déréguler le marché du travail. Le monopole d'embauche est définitivement aboli, l'action collective encore plus sévèrement encadrée, les protections accordées aux femmes et aux jeunes, parfois depuis le XIXe siècle, supprimées, et les wages councils démantelés ${ }^{14}$. L'État britannique joue le rôle d'employeur modèle dans cette dérégulation des rapports sociaux. Une vague de privatisations débute en 1984 avec la vente de British Telecom et affecte ensuite la plupart des entreprises nationales, jusqu'à British Rail qui est démantelée par étapes entre 1994 et 1997. Le gouvernement espère que la rigueur du marché s'imposera au sein des entreprises privatisées. Un modèle de gestion managérial est importé dans ce qui reste du service public. Des fonctionnaires doivent ainsi postuler pour leur propre travail désormais soumis à appel d'offre, et affronter la concurrence de prestataires privés. En 1991, la possibilité est offerte aux établissements du NHS de devenir des unités autonomes. Dans tous les cas, l'objectif est de briser les dynamiques collectives et d'affaiblir les syndicats encore très présents dans le secteur public ${ }^{15}$.

La réussite de ce projet de désarmement du mouvement syndical par la législation s'explique d'abord par son caractère progressif. Alors que le Trades Union Congress (TUC), la confédération des syndicats britanniques, avait pu lancer avec succès un vaste mouvement de résistance à l'Industrial Relations Act mis en œuvre par le gouvernement conservateur d'Edward Heath en 1971, et qui voulait pareillement encadrer l'activité syndicale, il est plus difficile de mobiliser les travailleurs contre des mesures qui, prises indépendamment, apparaissent partielles voire marginales ${ }^{16}$. Ensuite, les dispositions de ces lois successives sont présentées comme étant de simple bon sens, et le gouvernement bénéficie dans ce cadre du soutien d'une très large majorité de la presse quotidienne britannique. L'offensive politique de la droite thatchérienne s'accompagne en effet, dès la phase de conquête du pouvoir dans la seconde moitié des années 1970, d'une offensive médiatique qui vise à disqualifier symboliquement les syndicats en les présentant comme une entrave au bon fonctionnement de l'économie britannique, et une menace pour l'ordre social ${ }^{17}$.

Fort des encouragements d'une presse très majoritairement acquise à la cause

\footnotetext{
${ }^{13}$ Ibid., p. 301. Ces mesures se révèlent à double tranchant. Certes les dynamiques collectives de mobilisation et de passage à l'action sont sévèrement entravées par le passage à une démocratie de type indirect et représentatif, mais les votes à bulletin secret confèrent une nouvelle légitimité aux dirigeants syndicaux, et donnent plus de poids à une décision de faire grève.

${ }^{14}$ Ibid., pp. 322-325.

${ }^{15}$ Chris HOWELL, op. cit., pp. 153-155.

${ }^{16}$ Richard TAYLOR, op. cit., p. 284-285.

${ }^{17}$ Colin HAY, 'Narrating Crisis : The Discursive Construction of the 'Winter of Discontent", Sociology, vol. 30, no. 2, May 1996, pp. 253-277 ; Chris HOWELL, op.cit., pp. 143-144.
} 
conservatrice, le gouvernement de Margaret Thatcher met en œuvre, à côté de la stratégie législative, une stratégie politique de neutralisation du mouvement syndical dans laquelle on peut distinguer deux phases. Le gouvernement conservateur adopte tout d'abord une stratégie de marginalisation du TUC et des syndicats, qui vise à en saper l'autorité et la légitimité. Depuis la mise en place progressive des institutions de régulation collective des rapports sociaux par les gouvernements libéraux de la fin du XIX $\mathrm{X}^{\mathrm{e}}$ siècle, et plus encore depuis l'après-guerre et l'adoption d'un mode de gestion tripartite de l'économie dans le cadre d'un consensus autour d'une économie mixte, les syndicats britanniques sont en effet associés à l'élaboration des politiques gouvernementales, principalement dans les domaines économique et social.

Si les syndicats ne parviennent pas toujours à influencer les politiques publiques dans le sens qu'ils désirent, ils trouvent néanmoins dans les gouvernements de tous bords des interlocuteurs bienveillants. Ce mode de gestion de l'économie et les pratiques gouvernementales qui y sont associées changent avec l'arrivée de la droite conservatrice au pouvoir en 1979. Non seulement l'avis du TUC n'est plus sollicité par les ministres en charge des questions sociales et économiques, mais les instances de gestion tripartite de l'économie voient leurs attributions réduites, avant d'être progressivement abolies à partir du milieu des années $1980^{18}$. Cette stratégie d'exclusion et de délégitimation des syndicats connaît son épisode le plus marquant en 1984, lorsque le gouvernement interdit la présence des syndicats au Government Communication Headquarters (GCHQ), le service de renseignement électronique du gouvernement, au motif que l'organisation collective des travailleurs est incompatible avec la sécurité nationale ${ }^{19}$.

La seconde stratégie adoptée par le gouvernement conservateur est une stratégie d'affrontement et de répression, illustrée par la Grande Grève des mineurs de 1984-1985, laquelle oppose pendant plus d'un an le National Coal Board soutenu par le gouvernement à la National Union of Mineworkers (NUM), le syndicat des mineurs, qui rejette les fermetures de mines. Le gouvernement s'est préparé depuis plusieurs années à une confrontation dont il espère qu'elle lavera l'affront de 1974, lorsque le précédent gouvernement conservateur avait été défait par les mineurs. Des réserves de charbon ont été constituées et un important dispositif policier mis en place. Durant le conflit, de nombreux heurts opposent les mineurs à la police, résultant dans l'arrestation de plus de 11000 mineurs, dont 8000 sont poursuivis en justice. Une gigantesque opération de surveillance et de désinformation est montée par les services secrets britanniques, et les ressources de la NUM sont saisies par la justice. Face à cet assaut coordonné de toutes les composantes de l'État britannique, le TUC et les autres syndicats se refusent à apporter un soutien autre que symbolique aux mineurs. En dépit du soutien des communautés minières et d'une grande partie de l'opinion, la grève s'achève sur un échec ${ }^{20}$.

\footnotetext{
${ }^{18}$ C'est le cas des Industrial Training Boards, de la Manpower Servives Commission et du National Economic Development Council.

${ }^{19}$ J. WADDINGTON \& R. HOFFMANN (ed.), Trade Unions in Europe: facing challenges and searching for solutions, Bruxelles : European Trade Union Institute, 2000, p. 579.

${ }^{20}$ Kenneth O. MORGAN, Britain Since 1945 : The People's Peace, Oxford : Oxford University Press, 2001, pp. 472-475.
} 
Le rôle joué par l'arsenal législatif anti-syndical et l'offensive judiciaire et policière menée par l'État britannique dans l'affaiblissement du mouvement syndical fait l'objet de débats au sein de la communauté des historiens aussi bien que dans les rangs du mouvement syndical. Il a ainsi été affirmé que les politiques des gouvernements conservateurs n'avaient finalement fait que faciliter une reprise en main par les employeurs permise avant tout par la crise économique qui affecte l'économie britannique, et tout particulièrement le secteur manufacturier, à partir du milieu des années $1970^{21}$. Dans un contexte de montée du chômage, où le rapport de force au sein des entreprises devient moins favorable aux travailleurs, les licenciements et les fermetures d'usines désorganisent les bastions du mouvement syndical dans l'industrie manufacturière. Il n'en reste pas moins que les effets de la crise économique sur le secteur manufacturier ont été amplifiés par la politique monétaire du gouvernement conservateur qui privilégie une livre sterling forte et fait de la lutte contre l'inflation sa priorité, devant la lutte contre le chômage ${ }^{22}$. Quelque soit la part exacte jouée par ces différents facteurs, leur résultat est incontestable. En deux décennies, les effectifs syndicaux chutent de près de 40 pour cent. Si en 1979 13,3 millions de personnes appartenaient à un syndicat, elles ne sont plus que 7,6 millions en 2001, c'est-à-dire moins de 30 pour cent des travailleurs contre 55 pour cent en 1979. Seuls 35 pour cent des travailleurs sont couverts par un accord collectif sur les salaires et les conditions de travail. Le nombre de conflits sociaux diminue fortement pour atteindre, dans les années 1990, le nombre le plus faible jamais enregistré ${ }^{23}$.

\section{Les stratégies d'adaptation des organisations de travailleurs}

Prenant acte de cette nouvelle situation d'affaiblissement numérique, de désarmement économique et de disqualification symbolique, les organisations syndicales connaissent de profondes transformations à partir des années 1980. Elles développent notamment des stratégies plus élaborées en matière de gestion de leur image. Si dans les années 1970 la plupart des directions syndicales regardaient le champ médiatique comme accessoire par rapport au terrain social des luttes, et rares étaient celles, en dehors des syndicats d'employés, qui disposaient d'un véritable service de relations publiques, elles sont amenées à revoir leur stratégie dans ce domaine et à mettre en place des services plus professionnels de gestion de l'image. Le TUC les épaule en mettant à la disposition des syndicats qui lui sont affiliés des ressources en matière de communication et de relations publiques ${ }^{24}$. En 1989 , le succès de la grève des ambulanciers menée par les syndicats du secteur public - les mêmes qui s'étaient attirés l'opprobre des médias lors de l'«hiver du mécontentement $»-s$ 'explique notamment par une gestion judicieuse et collective de leur image par les syndicats. C'est la première fois que les syndicats gagnent la bataille de l'opinion publique et des médias face au gouvernement Thatcher. De manière plus anecdotique, cette revalorisation de l'image des syndicats est également passée par la participation à l'organisation de festivals, et par des

${ }^{21}$ Kenneth O. MORGAN, op. cit., p. 446 ; Chris HOWELL, op. cit., p. 159.

${ }^{22}$ Alastair J. REID, United We Stand : A History of Britain's Trade Unions, London : Penguin Books, 2005, pp. 399-400.

${ }^{23}$ Chris HOWELL, op. cit., p. 131.

${ }^{24}$ Alastair J. REID, op. cit., p. 411 ; J. WADDINGTON \& R. HOFFMANN (eds.), op. cit., p. 579. 
partenariats financiers avec des clubs de football. Ainsi la General Municipal and Boilermakers' Union (GMB) a-t-elle sponsorisé Luton, Brentford, Fulham et Woking $^{25}$. Ces partenariats culturels et sportifs visent à attirer les jeunes travailleurs vers le syndicalisme, et s'inscrivent donc aussi dans une politique de recrutement.

Les syndicats se sont en effet transformés pour endiguer la chute du nombre de leurs adhérents. Deux modèles organisationnels majeurs, qui tous deux ont été essayés par les syndicats britanniques, ont été identifiés par les commentateurs ${ }^{26}$. Un premier modèle, dit servicing model, repose sur la mise à la disposition des adhérents d'une offre de services accrus de la part du syndicat. Ainsi depuis le milieu des années 1980, de nombreux syndicats proposent à leurs adhérents des assurances, cartes de crédit, retraites complémentaires, bons d'achats, et autres services financiers. Pour inciter les adhérents à promouvoir le syndicat sur leur lieu de travail, le syndicat du secteur public, Unison, a choisi dans les années 1990 d'offrir des primes aux meilleurs recruteurs ${ }^{27}$. Dans ce premier modèle, l'accent est mis sur le professionnalisme du cadre de permanents qui gère cette offre de services, et donc sur une redynamisation de l'activité syndicale par le haut. Le deuxième modèle, connu sous le nom d'organising model, insiste quant à lui sur le renforcement de la base militante, et donc sur une redynamisation de l'activité syndicale par le bas. L'élargissement de la base militante passe par une campagne de recrutement agressive sur les lieux de travail, et donc par la formation d'un cadre de permanents spécialisés dans le recrutement. Sur le modèle d'initiatives similaires lancées par les confédérations américaine et australienne, le TUC a mis en place en 1998 une Organising Academy chargée de la formation des futurs agents recruteurs, dans le cadre d'un projet intitulé «nouveau syndicalisme» (new unionism) ${ }^{28}$. En 2008, le TUC affirmait que ce programme avait permis de recruter 50000 nouveaux adhérents pour les syndicats britanniques et 4500 militants syndicaux, et de former 270 permanents spécialisés dans le recrutement. Fort de ce bilan, le TUC a décidé de lancer une Activist Academy, qui se donne pour objectif de former 1000 nouveaux permanents en trois ans ${ }^{29}$.

Il est cependant notable que les campagnes de recrutement, si elles permettent de renforcer le taux de syndicalisation dans des entreprises et des secteurs où les syndicats sont déjà bien implantés, peinent à créer une dynamique de syndicalisation sur les lieux de travail où les syndicats sont absents, ce qui est notamment le cas dans le secteur privé des services. L'ouverture vers de nouveaux secteurs et de nouveaux types de salariés est pourtant une nécessité pour les syndicats, du fait de l'érosion de leurs bastions traditionnels dans les régions industrielles du nord de

${ }^{25}$ J. WADDINGTON \& R. HOFFMANN (ed.), op. cit., p. 63.

${ }^{26}$ K. BRONFENBRENNER, F. FRIEDMAN, R. HURD, R. OSWALD \& R. SEEBER (ed.). Organizing to Win: New Research on Union Strategies, Ithaca: New York, 1998, p. 43.

${ }^{27}$ Ibid., p. 64

${ }^{28}$ E. HEERY, M. SIMMS \& D. SIMPSON, Research Bulletin : New Unionism Research Project, No. 5, June 1999, Cardiff University. Voir aussi le site du TUC qui présente le projet new unionism : http://www.tuc.org.uk/theme/index.cfm?theme=newunionism (consulté le 22 janvier 2009).

${ }^{29}$ Trades Union Congress, 'Unions celebrate $10^{\text {th }}$ anniversary of TUC Organising Academy', cf. http://www.tuc.org.uk/organisation/tuc-15476-f0.cfm?theme=newunionism (consulté le 22 janvier 2009). 
l'Angleterre, de l'ouest de l'Écosse et du Pays de Galles, et de la croissance explosive des activités de service dans le sud-est de l'Angleterre et l'est de l'Écosse. Les destructions et les créations d'entreprises induites par ce déplacement du centre de gravité économique, qui ont souvent été l'occasion pour les employeurs d'imposer un rapport de force et des conditions de travail moins favorables à leurs salariés, ont parfois aussi stimulé chez ces derniers un besoin d'organisation collective. Les syndicats cherchent donc à étendre leur recrutement dans ces secteurs, d'un côté vers les travailleurs bien rémunérés spécialisés dans la finance ou les technologies de l'information, et de l'autre côté vers les travailleurs précaires, souvent employés sur la base de contrats flexibles et temporaires ${ }^{30}$. De même, l'organisation des travailleurs originaires de l'immigration, souvent en butte à l'hostilité de l'État et exclus des dispositifs de protection accordés par la loi, constitue un terrain d'expansion possible pour les syndicats. On peut citer ici l'exemple de la campagne Justice for Cleaners, menée par la Transport and General Workers Union (TGWU) sur le modèle d'une initiative similaire aux États-Unis. Cette campagne vise à organiser collectivement les employés des sociétés de ménage chargés du nettoyage des bureaux dans les quartiers d'affaires de Londres. Grâce à Justice for Cleaners, ces travailleurs précaires majoritairement issus de l'immigration ont réussi à obtenir en 2006 et 2007 des concessions de la part des sociétés de ménage et des grands groupes financiers qui louent leurs services ${ }^{31}$. Cette politique d'ouverture s'est également traduite par des réformes de l'organisation interne des syndicats. Au sein du TUC, des groupes comme les femmes et les minorités, longtemps ignorés dans un mouvement syndical dominé par des syndicats à dominante masculine et blanche, ont vu leur place progressivement reconnue. Des dispositifs ont été mis en place au sein des instances de décision pour assurer la représentation de ces différents groupes. Il en va de même au sein de la plupart des syndicats. Le fonctionnement du TUC a également évolué pour tenir compte du déplacement du centre de gravité du mouvement syndical vers les syndicats du secteur public qui, longtemps subordonnés à ceux des secteurs industriels traditionnels - mines, industrie manufacturière, transport -, dictent aujourd'hui les priorités du TUC.

Ces mutations organisationnelles profondes se sont déroulées dans un cadre général de recomposition du paysage syndical britannique. Les fusions, déjà nombreuses dans les années 1960 et 1970, se sont accélérées depuis les années 1980. On passe de 454 syndicats en 1979 à 233 en 1998. Les deux grandes tendances sont l'expansion des syndicats généralistes, et la constitution de grands syndicats sectoriels $^{32}$. Les commentateurs, comme les syndicalistes, sont partagés sur le bilan

${ }^{30}$ Alastair J. REID, op. cit., pp. 407-409 ; J. WADDINGTON \& R. HOFFMANN (ed.), op. cit., p. 615.

${ }^{31}$ Heather STEWART, 'Dirty business : cleaning up the Square Mile's mess', Observer, 17 décembre 2006. Cf. aussi le site de la TGWU, http://www.tgwu.org.uk, 'Campaigning'

32 J. WADDINGTON \& R. HOFFMANN, op. cit., p. 591 ; Alastair J. REID, op. cit., p. 411. La General and Municipal Workers Union (GMWU) s'allie à l'Amalgamated Society of Boilermakers Shipwrights Blacksmiths and Structural Workers (ASBSBSW), le syndicat des ouvriers des chantiers navals, à l'Association of Professional Executive Clerical and Computer Staff (APEX), regroupant les employés de bureau du secteur privé, et la National Union of Tailors and Garment Workers (NUTGW) - le syndicat du secteur de l'habillement pour former en 1982 la General, Municipal, Boilermakers and Allied Trade Union (GMB). 
de cette vague de fusions-acquisitions. Si Alastair J. Reid suggère que la constitution de grands syndicats sectoriels leur donne un poids plus important dans les négociations avec les employeurs, Jeremy Waddington souligne que les fusions ne vont pas sans poser des problèmes organisationnels au sein des syndicats recomposés. Une réorganisation interne profonde se révèle en effet souvent nécessaire pour tenir compte de l'hétérogénéité des groupes représentés. La taille croissante des syndicats pose également des problèmes en matière de représentativité et de cohésion au sein de l'organisation. Le caractère souvent défensif de ces alliances a été souligné. Quant il ne s'est pas agi d'annexions pures et simples de petites organisations en perte de vitesse par des syndicats plus puissants, ce sont souvent des organisations affaiblies qui ont joint leur force, escomptant des bénéfices financiers d'une restructuration interne, et aspirant à préserver leur influence grâce à leur poids numérique retrouvé.

\section{Le repositionnement politique et institutionnel des syndicats britanniques}

Créer un rapport de force favorable aux organisations de travailleurs est une question non seulement de modèle organisationnel, mais aussi de positionnement politique. Ne bénéficiant plus du statut d'institution quasi-étatique associée aux décisions gouvernementales, le mouvement syndical a négocié de nouvelles relations avec les gouvernements, les institutions étatiques britanniques et européennes, ainsi que les autres acteurs de la scène sociale. Pendant le premier mandat du gouvernement Thatcher (1979-1983), le mouvement syndical adopte pourtant la même politique de non-coopération qui avait réussi à faire échouer la précédente tentative d'un gouvernement conservateur d'encadrer l'action collective par la loi. Lorsque le gouvernement d'Edward Heath avait voulu mettre en œuvre son Industrial Relations Act en 1971-1972, il s'était heurté à une résistance syndicale organisée et coordonnée par le TUC. D'abord passive, cette résistance était devenue active pendant l'été 1972, suite notamment à l'emprisonnement de cinq dockers pour non-respect des dispositifs prévus par la législation conservatrice. Le gouvernement Heath avait dû renoncer à faire appliquer l'Industrial Relations Act, qui avait été aboli dès le retour au pouvoir des travaillistes en 1974. Dans les premières années de gouvernement Thatcher, le TUC comme l'ensemble du

En 1992, l'Amalgamated Engineering Union (AEU) et l'Electrical, Electronic, Telecommunications and Plumbing Union (EETPU), deux syndicats qui regroupaient à l'origine exclusivement des ouvriers qualifiés du secteur manufacturier, fusionnent pour former le syndicat à vocation généraliste l'Amalgamated Engineering and Electrical Union (AEEU). Cette dimension généraliste s'est encore accentuée avec l'alliance avec le syndicat Manufacturing, Science and Finance (MSF) qui a résulté dans la création de Amicus en 2001, puis la fusion avec la TGWU, qui a pris effet en 2007. Le super-syndicat formé par cette alliance, Unite, regroupe près de 2 millions de travailleurs. Des projets de fusion sont en cours avec le syndicat nord-américain United Steelworkers pour former une organisation internationale, Workers Uniting, qui compterait plus de 3 millions d'adhérents. À côté de la formation de ces deux grandes centrales généralistes, des fusions en série ont abouti à la formation de grands syndicats sectoriels dans les médias en 1992, le secteur public en 1993, les communications en 1995 et l'administration en 1998 avec respectivement la Graphical Paper and Media Union (GPMU), Unison, la Communication Workers Union (CWU) et la Public and Commercial Services Union (PCS). 
mouvement syndical choisissent pareillement d'opposer une résistance passive à la législation anti-syndicale mise en œuvre par les conservateurs. Pour les syndicalistes comme pour les travaillistes, un gouvernement dont la politique sociale est en contradiction flagrante avec la recherche de paix sociale qui a prévalu dans la gestion de l'économie et des rapports sociaux depuis l'après-guerre, court inévitablement à sa perte. Les prochaines élections amèneraient nécessairement le retour des travaillistes et d'une gestion plus consensuelle des questions économiques et sociales ${ }^{33}$.

Face à un Parti travailliste affaibli par la défection de son aile droite qui forme le Social Democratic Party en 1981, et dans le contexte de patriotisme exacerbé qui suit la guerre des Malouines de 1982, les conservateurs sont réélus en 1983. Le mouvement syndical doit prendre acte de l'ancrage du projet thatchérien et de la consolidation de sa base populaire. La défaite des mineurs, lors de la grève qui les oppose à leurs employeurs soutenus par le gouvernement en 1984-1985, finit de discréditer toute stratégie de confrontation avec le gouvernement. Au milieu des années 1980, le TUC abandonne majoritairement sa politique de résistance passive face au gouvernement Thatcher, et adopte une nouvelle orientation politique, dite néo-réaliste (new realism). Cette politique de coopération est cependant vouée à l'échec, face à un gouvernement dont le traitement policier de la grève des mineurs et la décision concernant le GCHQ montrent qu'il regarde le mouvement syndical comme 1' «ennemi intérieur ${ }^{34}$. L'hostilité du gouvernement conservateur, son offensive législative et son refus de reconnaître une quelconque légitimité politique au TUC permettent cependant au mouvement syndical de se livrer à un exercice de réévaluation salutaire. L'illusion d'une place assurée du mouvement syndical parmi les institutions séculaires de l'État britannique vole en éclat. L'analyse, partagée par la plupart des commentateurs à la fin des années 1970, selon laquelle tout gouvernement doit composer avec le mouvement syndical dans la formulation et l'application de sa politique économique, est ébranlée par l'intransigeance du gouvernement de Margaret Thatcher. La thèse d'un avenir néo-corporatiste pour la Grande-Bretagne est invalidée par les pratiques gouvernementales des conservateurs au pouvoir ${ }^{35}$. Ce cadre intellectuel pluraliste d'analyse des rapports sociaux, produit de l'économie mixte de l'après-guerre et qui avait fini par se naturaliser en une doxa acceptée par les syndicalistes comme par les commentateurs, fondait également l'idée que les travailleurs négociaient leur salaire et leurs conditions de travail avec leur employeur sans aucune intervention de l'État, thèse connue sous les noms de volontarisme (voluntarism) ou de collective laissez-faire. L'hypothèse volontariste soutenait l'illusion d'une puissance autonome des syndicats, capables d'obtenir par la seule force de leur poids économique et numérique des concessions de la part des employeurs, et occultait le rôle joué par l'État britannique dans la constitution et la consolidation du système de régulation collective des rapports sociaux depuis la fin

${ }^{33}$ Richard TAYLOR, op. cit., p. 268.

${ }^{34}$ Margaret Thatcher aurait déclaré devant les parlementaires conservateurs le 19 juillet 1984 : 'We had to fight an enemy without in the Falklands. We always have to be aware of the enemy within, which is more difficult to fight and more dangerous to liberty.' Voir J. KELLY \& B. ANGELO, 'A Long Summer of Discontent', Time, 30 juillet 1984.

${ }^{35}$ Colin CROUCH, The Politics of Industrial Relations, London: Fontana, 1979, pp. 190-191. 
du XIX $^{\mathrm{e}}$ siècle $^{36}$. Face à la révolution impulsée dans les rapports sociaux par l'offensive conservatrice, les syndicalistes doivent constater que les possibilités d'organisation et d'action collectives ne dépendent pas de leur seule force, mais sont aussi déterminées par un environnement politique, institutionnel et judicaire façonné par l'État britannique.

À partir de ce constat, le mouvement syndical adopte de nouvelles stratégies politiques dès le milieu des années 1980. Privé de sa place au cœur des institutions de l'État britannique, le TUC prend acte du rôle de groupe de pression auquel il est relégué, et réintègre un mouvement social recomposé, aspirant à prendre la tête d'une coalition d'organisations non-gouvernementales dont les objectifs rejoignent les siens. Ainsi le TUC s'associe-t-il aux campagnes menées par la Consumers' Association, la National Association of Pension Funds, Help the Aged ou encore Stonewall $^{37}$. Modérant son adhésion à la thèse volontariste et son hostilité traditionnelle à toute législation positive concernant les rapports sociaux, le mouvement syndical opère pareillement un tournant législatif. L'absence d'un droit de grève ancré positivement dans le droit a permis au gouvernement conservateur de priver le mouvement syndical de ses armes. Aussi, à partir de la seconde moitié des années 1980, le TUC fait campagne pour l'inscription dans la loi d'un éventail de droits protégeant les travailleurs, leurs conditions de travail et leur organisation collective. Ce changement d'orientation s'explique aussi par le poids croissant au sein du TUC des syndicats du secteur public, porteurs depuis longtemps de ces revendications. C'est notamment le cas du salaire minimum, dont la mise en place est placée au cœur du programme de réformes sociales désormais porté par le TUC.

Dans un contexte politique hostile au niveau national, le mouvement syndical se tourne vers l'Europe pour jeter les bases d'un tel droit positif. Ce tournant proeuropéen, pris dans la seconde moitié des années 1980, constitue pareillement un repositionnement radical du mouvement syndical britannique. Dans les années 1970, les syndicats avaient en effet fait campagne contre l'adhésion de la Grande-Bretagne à la Communauté Economique Européenne (CEE), puis en faveur du «non » dans le référendum sur l'appartenance à la CEE organisé en 1975 par le gouvernement travailliste d'Harold Wilson. La CEE apparaissait alors aux organisations de travailleurs comme un club capitaliste hostile aux intérêts de la classe ouvrière britannique. À partir des années 1980, le mouvement syndical révise son jugement sur la CEE, et se tourne vers les directives et la justice européennes pour atténuer les effets de la dérégulation du marché du travail, et trouver dans les droits individuels conférés aux salariés par la législation européenne une contrepartie à l'effondrement du système britannique de régulation collective des conditions de travail. Le TUC installe une officine à Bruxelles, et réserve à un accueil chaleureux à Jacques Delors, alors Président de la Commission Européenne, lors de son Congrès annuel de $1988^{38}$.

${ }^{36}$ Chris HOWELL, op. cit., pp. 5-6.

${ }^{37}$ J. WADDINGTON \& R. HOFFMANN, op. cit., p. 606 ; Edmund HEERY, 'The Relaunch of the Trades Union Congress', British Journal of Industrial Relations, Vol. 36, No. 3, 1998, pp. 339-360.

${ }^{38}$ Chris HOWELL, op. cit., pp. 181-182 ; J. WADDINGTON \& R. HOFFMANN, op. cit., p. 602. 
Des éléments de ce programme législatif sont réalisés suite au retour des travaillistes au pouvoir en 1997. La mise en place d'un salaire minimum est votée par le Parlement en 1999. Cependant, sous la pression du patronat, la commission sur les bas salaires (Low Pay Commission) chargée de l'élaboration du dispositif fixe son niveau très bas. Par la suite, le gouvernement intervient pour diluer plus encore les effets du salaire minimum, au nom de la flexibilité du marché du travail. Pareillement, l'Employment Relations Act de 1999 doit faciliter la reconnaissance des syndicats au sein de l'entreprise, mais sa mise en œuvre et son impact sur les rapports sociaux au sein de l'entreprise sont délibérément restreints par le gouvernement néo-travailliste ${ }^{39}$. Enfin, conformément à ses promesses de campagne, le gouvernement Blair accepte en 1997 de signer le volet social du traité de Maastricht, auquel le gouvernement Major avait obtenu que le Royaume-Uni puisse se soustraire, ainsi que la directive européenne sur le temps de travail.

\section{Les syndicats face à la modernisation du Parti travailliste}

Cette mise en œuvre minimale et tronquée par le gouvernement travailliste du programme de réformes porté par le mouvement syndical reflète la transformation profonde de la relation entre les syndicats et le Parti travailliste qui, commencée dans les années 1980, s'accélère dans les années 1990. Les syndicats sont en effet les principales victimes politiques et symboliques de l'entreprise de modernisation et de «droitisation» progressive menée par les directions successives du Parti travailliste. La fin des années 1970, marquée par une polarisation de la vie politique britannique, ne laissait pourtant rien présager d'une telle transformation du Parti travailliste et de son attitude vis-à-vis du mouvement syndical. De même que l'aile droite impose alors son programme d'ordre et d'orthodoxie économique au Parti conservateur, l'aile gauche acquiert une influence croissante au sein du mouvement ouvrier, à la fois dans les syndicats et au sein du Parti travailliste. La radicalisation du parti est illustrée par l'élection de Michael Foot comme leader en 1980, et par la bataille à couteaux tirés pour le poste de leader-adjoint, entre l'aile gauche et l'aile droite du parti, rassemblées respectivement derrière Tony Benn et Denis Healey. Face aux politiques thatchériennes d'austérité économique, les syndicats et la base du parti mettent en avant des politiques de relance de l'économie et d'aide à l'emploi. Le manifeste du parti pour les élections de 1983 attaque de manière virulente le bilan du gouvernement Thatcher, et détaille un programme d'action d'urgence complètement à rebours des politiques conservatrices. Suite à la victoire des conservateurs, l'aile droite du parti attribue la défaite à ce programme, jugé trop radical, sur lequel les travaillistes ont fait campagne. Le centriste Neil Kinnock, qui remplace Michael Foot à la tête du parti, lance un programme dit de modernisation. Le Parti travailliste prend acte de la nouvelle donne politique établie par les conservateurs. Les syndicats, montrés du doigt en raison de leur caractère rétrograde et radical supposé, sont accusés de faire du tort au parti. Aussi la nouvelle direction travailliste prend-elle ses distances avec le mouvement syndical, et se montre particulièrement discrète lors du conflit qui oppose la NUM au National Coal Board soutenu par le gouvernement. Les nouvelles défaites aux élections de 1987 et 1992 renforcent dans l'aile droite du parti la conviction que la restauration des fortunes

${ }^{39}$ Chris HOWELL, op. cit., p. 180 ; J. WADDINGTON \& R. HOFFMANN, op. cit., p. 603. 
électorales du parti passe par une rupture avec les politiques et les valeurs avec lesquelles les travaillistes sont traditionnellement associés. Aussi le nouveau rapport de forces social instauré par les conservateurs ne doit-il pas être remis en cause. Peter Mandelson écrit ainsi à propos du projet néo-travailliste, dont il est un des principaux promoteurs :

Sa stratégie consiste à partir de là où Margaret Thatcher s'est arrêtée, plutôt qu'à démanteler tout ce qu'elle a fait ${ }^{40}$. [...] La transformation des rapports sociaux en Grande-Bretagne a été un progrès, et les dispositifs légaux mis en place par les conservateurs pour les encadrer resteront en place. ${ }^{41}$

Pour être en mesure de faire concurrence aux conservateurs sur leur propre terrain, il est nécessaire aux modernisateurs néo-travaillistes de repositionner le parti comme un parti centriste favorable à l'entreprenariat privé, et donc de briser l'association symbolique avec les syndicats. On peut mesurer le chemin parcouru depuis les années 1970 en comparant les programmes électoraux de 1979 et 1997. Alors que le «soutien entier du TUC » est présenté comme la garantie de la mise en œuvre des politiques sociales et économiques annoncées par le 1979 Labour Party Manifesto, et que celui-ci réaffirme la nécessaire «coopération entre le gouvernement, les syndicats et les directions d'entreprises ${ }^{42}$, les syndicats occupent une place très différente dans le programme New Labour : Because Britain Deserves Better de 1997. Loin d'insister sur la coopération étroite avec le mouvement syndical, ce programme multiplie les garanties d'une totale indépendance à l'égard des syndicats.

Nous avons réécrit notre constitution, autour d'un nouvel article 4, pour souligner notre attachement à l'entreprise tout autant que notre attachement à la justice. Nous avons modifié la façon dont nous élaborons nos politiques, et modernisé nos relations avec les syndicats, qui savent qu'ils peuvent attendre de la part d'un gouvernement travailliste une attitude équitable, mais pas de traitement de faveur. Nos députés sont désormais sélectionnés par les membres ordinaires du parti, et non par des petits comités ou des groupes de pression. [...] Dans le domaine des rapports sociaux, nous indiquons clairement qu'il n'y aura pas de retour aux piquets volants, aux blocages d'entreprises tierces, aux grèves non précédées d'un vote, ou au cadre légal qui prévalait dans les années 1970. Au lieu de cela, les salariés bénéficieront individuellement de droits élémentaires sur leur lieu de

\footnotetext{
40 'Its strategy is to move forward from where Margaret Thatcher left off, rather than to dismantle every single thing she did.' (Peter MANDELSON, The Blair Revolution Revisited, Westminster: Politico's Publishing, 2002, p. 1).

41 'British industrial relations has been changed for the better, and its basic legal framework which the Conservatives established will remain in place [...].' Ibid., pp. 12-13.

42 'Now, with the renewed cooperation of the trade union movement, Labour will continue the battle against rising prices. With the wholehearted backing of the TUC, we have set ourselves a new target, to get inflation down to 5 per cent by 1982. [...] This is a positive strategy for industry, based on cooperation between Government, trade unions, and management.', Labour Party, 1979 Labour Party Manifesto, London : Labour Party, 1979.
} 
travail, où notre objectif est une relation partenariale, et non conflictuelle, entre employeurs et employés. ${ }^{43}$

C'est non seulement le lien symbolique avec les syndicats qui est remis en cause, mais également le lien organisationnel et ombilical avec le mouvement syndical. Philip Gould, qui conseille la nouvelle direction blairiste du parti dans les années 1990, voit dans les syndicats, qu'il présente comme des organisations archaïques à peine sorties de l'ère victorienne, le principal obstacle à la nécessaire modernisation du parti.

Le Parti travailliste fut dès sa naissance un parti conservateur. Il était trop lié au mouvement syndical, trop obsédé par le secteur public, trop enferré dans sa mythologie, trop ancré dans le passé ${ }^{44}$. [...] Bénéficiant d'une croissance numérique rapide et attirant des milliers d'ouvriers, les syndicats possédaient une force et une intelligence qui faisaient défaut aux autres organisations du mouvement ouvrier. Mais, produits d'avancées prudentes et défensives, ils furent aussi à l'origine de la rigidité organisationnelle et des blocs de voix. Plus préoccupés par leurs seuls intérêts et par eux-mêmes que leurs homologues continentaux, les syndicats britanniques trouvaient leur modèle dans l'artisanat et non l'industrie, et avaient été éprouvés par la législation hostile des années 1890 et de 1906. Ils n'étaient pas des institutions intrinsèquement modernisatrices. ${ }^{45}$

Pareillement, la vision du Parti travailliste comme organe de représentation politique des intérêts collectifs et des aspirations communes du mouvement ouvrier, et la centralité des syndicats dans son histoire, sont niées. Peter Mandelson souligne que « dès les premières années de l'existence du parti, les dirigeants travaillistes ont toujours insisté sur le fait que le Parti travailliste était un parti national, et non pas

43 'We have rewritten our constitution, the new Clause IV, to put a commitment to enterprise alongside the commitment to justice. We have changed the way we make policy, and put our relations with the trade unions on a modern footing where they accept they can get fairness but no favours from a Labour government. Our MPs are all now selected by ordinary party members, not small committees or pressure groups. [...] In industrial relations, we make it clear that there will be no return to flying pickets, secondary action, strikes with no ballots or the trade union law of the 1970s. There will instead be basic minimum rights for the individual at the workplace, where our aim is partnership not conflict between employers and employees.', Labour Party, New Labour : Because Britain Deserves Better, London : Labour Party, 1997.

44 'Labour was born a conservative party: it was too close to trade unionism; too obsessive about public ownership; too tied to myth; too rooted in the past.', Philip GOULD, The Unfinished Revolution: How the Modernisers Saved the Labour Party, London: Abacus 1999, p. 24.

${ }^{45}$ 'Growing rapidly in numbers, attracting thousands of working-class people, trade unions had a robustness and common sense which the other Labour organisations lacked. But they were also the source of organisational rigidity and the block vote, and they were born of cautious, defensive progress. By nature more sectional and inward-looking than their continental cousins, British trade unions were rooted in crafts not industry, battered by hostile legislation in the $1890 \mathrm{~s}$ and 1906. They were not intrinsically modernising institutions.', Ibid., p. 25. 


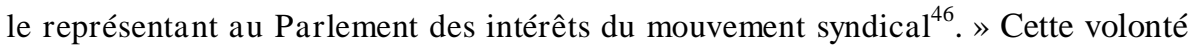
de diminuer le rôle des syndicats au sein du parti se traduit par une réduction progressive du poids des syndicats dans les instances décisionnelles du parti. Leur part des voix à la conférence annuelle du parti passe de $90 \%$ à $70 \%$ en 1993, puis à $50 \%$ en 1995. Le système des blocs de voix, qui conférait poids et cohésion au mouvement syndical au sein du parti, est aboli au profit du scrutin individuel ${ }^{47}$. La direction du parti cherche pareillement à réduire la dépendance financière du parti à l'égard des syndicats. En 1983, ce ne sont en effet pas moins de 96\% des revenus du parti qui proviennent des contributions syndicales. La professionnalisation de la collecte des cotisations individuelles permet de réduire cette proportion à $40 \%$ au milieu des années 1990, puis à $30 \%$ en 2002 avant d'atteindre le plancher de 22,8\% en $2005^{48}$. À côté des cotisations des adhérents et sympathisants, le parti fait appel depuis 1996 à de riches mécènes pour assurer une part croissante de son financement. Le système de sponsoring des parlementaires par les syndicats est pareillement aboli, et l'accent mis sur la participation individuelle des syndicalistes à la vie du parti et à l'élaboration des politiques gouvernementales. Ce ne sont plus les représentants des syndicats qui sont invités à siéger dans les commissions mises en place par le gouvernement néo-travailliste, mais des syndicalistes choisis pour leur loyauté individuelle au gouvernement. Toutes ces réformes organisationnelles tendent à neutraliser les dynamiques collectives au sein du Parti travailliste et la constitution d'un front syndical uni d'opposition à la direction du parti. De leur côté, les syndicats continuent de soutenir le Parti travailliste, en dépit des efforts faits par le gouvernement conservateur dans les années 1980 pour rompre le lien historique entre les deux ailes du mouvement ouvrier. Le Trade Union Act de 1984 prévoyait que le financement du parti par les syndicats fasse l'objet d'un vote tous les dix ans au sein de chaque organisation. Mais en 1985-1986 et à nouveau en 1995-1996 et en 2003-2004, les syndicats affiliés ont confirmé leur soutien financier au Parti travailliste. Par ailleurs, les syndicats continuent de fournir l'essentiel des ressources militantes pour les campagnes électorales du Parti travaillistes ${ }^{49}$. Depuis 1994, le lien entre le parti et les syndicats qui y sont affiliés a même été renforcé par la mise en place de la Trade Union and Labour Party Liaison Organisation (TULO), qui non seulement coordonne le soutien organisationnel et financier des syndicats au parti en période électorale, mais formalise également les communications entre le parti et les syndicats qui y sont affiliés ${ }^{50}$.

\footnotetext{
46 'From the party's earliest days, Labour's leaders were always keen to emphasise that Labour was a national party, not the representative in Parliament of trade-union sectional interests.' Peter MANDELSON, op. cit., p. 24.

${ }^{47}$ Florence FAUCHER-KING, 'La "modernisation" du Parti travailliste, 1997-2007. Succès et difficultés de l'importation du modèle entrepreneurial dans un parti politique', Politix, vol. 21, n०81, 2008, p. 136.

${ }^{48}$ Carole BACHELOT, 'Parti socialiste français et Parti travailliste britannique. Le cas des groupes dirigeants', Vingtième Siècle. Revue d'Histoire, n 96 , octobre-décembre 2007, p. 119 ${ }^{49}$ J. WADDINGTON et R. HOFFMANN, op. cit., p. 599.

${ }^{50}$ TULO rassemble début 2009 l' Associated Society of Locomotive Steam Enginemen and Firemen (ASLEF), la Broadcasting Entertainment Cinematograph and Theatre Union (BECTU), la Bakers Food and Allied Workers Union (BFAWU), Community, la CWU, la GMB, la Musicians Union (MU), la National Association of Colliery Overmen Deputies and Shotfirers (NACODS), la NUM, la Transport Salaried Staffs' Association (TSSA), la Union
} 


\section{Les alliés loyaux et dociles du néo-travaillisme?}

Les syndicats n'ont pas seulement été des victimes consentantes de la minoration de leur rôle au sein du parti. Nombre de syndicalistes ont en effet été les soutiens, sinon les acteurs et les promoteurs du projet néo-travailliste. Ainsi Tom Sawyer, qui, en tant que secrétaire général du Parti travailliste de 1994 à 1998 est un des principaux artisans des réformes néo-travaillistes au sein du parti, est un syndicaliste, ancien vice-secrétaire général de la National Union of Public Employees (NUPE). Présentant à l'exécutif du parti un projet de refonte organisationnelle sous la forme d'un Business Plan dix semaines après son entrée en fonction $^{51}$, il procède à la professionnalisation du personnel du parti, à la centralisation de son organisation et à la mise au pas des foyers de contestation au sein du parti. Il préside également à la suppression dans la constitution du parti du fameux article 4 (Clause IV), qui fixait comme objectif au parti la redistribution de la richesse produite par la socialisation des moyens de production, de distribution et d'échange. On pourra pareillement souligner le rôle d'anciens syndicalistes dans les gouvernements Blair et Brown, notamment John Prescott, ancien permanent de la National Union of Seamen (NUS) et Vice-Premier Ministre de 1993 à 2007, ou encore Alan Johnson, Secrétaire Général de la Union of Communication Workers (UCW) de 1992 à 1993 puis de la Communication Workers Union (CWU) de 1993 à 1997, et qui depuis s'est progressivement élevé dans la hiérarchie gouvernementale. John Prescott, soutien inconditionnel de Tony Blair pendant ses dix années au pouvoir, a ainsi été accusé par les adversaires du projet néo-travailliste au sein du parti de servir de caution ouvrière aux politiques blairistes d'inspiration néo-libérale.

Par delà ces exemples individuels d'adhésion à la refonte néo-travailliste du parti prônée par Tony Blair et en écho à celle-ci, le TUC s'est fait dans les années 1990 le porte-parole d'un néo-syndicalisme, notamment sous la direction de son secrétaire général John Monks (1993-2003). Le concept central du programme néotravailliste en matière de rapports sociaux, et autour duquel s'articule le projet néosyndicaliste, est celui de partenariat (partnership) ${ }^{52}$. Si les architectes de la troisième voie blairiste aiment à définir cette idée de partenariat social à partir des modèles rhénans et scandinaves de démocratie sociale et de régulation collective, le social partnership néo-travailliste possède en vérité peu de points communs avec ceux-ci. Un élément essentiel des modèles rhénan et scandinave est en effet l'existence de syndicats forts, associés à la gestion à long terme des entreprises. Or le gouvernement néo-travailliste ne souhaite ni remettre en cause la législation conservatrice pour renforcer l'organisation syndicale, ni donner aux syndicats un rôle décisionnel au sein de l'entreprise pour contrebalancer la vision à court terme des investisseurs financiers, ni même restaurer les institutions nationales de régulation collective des salaires et des conditions de travail qui existaient jusqu'aux années 1980. Le modèle britannique est donc plus proche des modèles français et américains, où les droits individuels conférés aux salariés visent à compenser la

of Construction Allied Trades and Technicians (UCATT), Unison, Unite, Unity et la Union of Shop Distributive and Allied Workers (USDAW).

${ }^{51}$ Florence FAUCHER-KING, op. cit., p. 132.

${ }^{52}$ Chris HOWELL, op. cit., p. 177. 
faiblesse de l'organisation collective ${ }^{53}$. La notion de partenariat telle qu'elle est envisagée par les néo-travaillistes vise plutôt à encourager la paix sociale en privilégiant une résolution consensuelle des conflits au sein de l'entreprise. Ainsi l'Employment Act de 2002, en plus d'accorder de nouveaux droits individuels aux travailleurs en matière de congés et d'activités syndicales, met-il en place des dispositifs au sein des entreprises afin d'éviter que les différends entre employeurs et salariés finissent devant une cour de justice.

Les syndicats ont certes obtenu quelques avancées symboliques. L'interdiction des syndicats au GCHQ est levée dès l'arrivée des travaillistes au pouvoir en 1997. Les employés du MI6 et du MI5 - les services secrets britanniques - obtiennent à leur tour le droit d'être représentés par des syndicats, respectivement en 2001 et $2008^{54}$. À côté de ces concessions mineures, la volonté du gouvernement travailliste de ne pas remettre en cause les grandes lignes des lois anti-syndicales, et la poursuite de politiques d'inspiration néo-libérale, provoquent rapidement des réactions hostiles de la part des syndicats, notamment dans les services publics. Dès 1999, Ken Cameron, alors secrétaire général de la Fire Brigades Union (FBU), le syndicat des pompiers, n'hésite pas à critiquer le gouvernement travailliste lors de la conférence annuelle du TUC. Il va même jusqu'à suggérer qu'une séparation des deux ailes du mouvement ouvrier leur serait bénéfique, en permettant aux syndicats d'adopter une attitude véritablement critique et constructive vis-à-vis du gouvernement $^{55}$. En 2002, le même syndicat, désormais dirigé par Andy Gilchrist, mène une grève de plus de six mois pour obtenir une augmentation de salaire de 40 pour cent. L'intransigeance du gouvernement et la défaite des pompiers conduit la FBU a se désaffilier du Parti travailliste en 2004. En 2003, plusieurs sections écossaises de la très militante Rail, Maritime and Transport Union (RMT) décident de soutenir financièrement le Scottish Socialist Party (SSP), un parti de gauche radicale formé par des Trotskystes et des déçus du Parti travailliste, ce qui conduit en 2004 le Parti travailliste à désaffilier la RMT. Ce renouveau militant est également marqué par l'élection de dirigeants issus de l'aile gauche (broad left) du mouvement syndical, vite surnommés «The Awkward Squad» par leurs adversaires $^{56}$. Â la conférence annuelle du Parti travailliste de 2003, les dirigeants des syndicats les plus puissants - TGWU, Amicus, GMB - mènent une offensive musclée contre les politiques du gouvernement Blair en matière de retraites, de financement des services publics et de soutien à l'industrie manufacturière. Si certains des dirigeants syndicaux de l' «Awkward Squad» ont été tentés un temps par une rupture avec le Parti travailliste, en faveur par exemple d'un soutien politique et financier à des partis de gauche radicale comme la Respect Coalition ou le SSP, cette perspective s'est aujourd'hui éloignée avec l'éclipse de ces forces

${ }^{53}$ Ibid., pp. 187-188.

${ }^{54}$ David HENCKE, 'MI5 opens door for union to represent spies', Guardian, 12 September 2008.

${ }^{55}$ J. WADDINGTON \& R. HOFFMANN, op. cit., p. 600.

${ }^{56}$ Le terme est apparu autour de 2002-2003. Les membres de cet 'Awkward Squad' étaient alors Bob Crow (RMT), Jeremy Dear (National Union of Journalists), Billy Hayes (Communication Workers Union), Paul Mackney (National Association of Teachers in Further and Higher Education, aujourd'hui partie de la University and College Union), Mark Serwotka (Public and Commercial Services Union), Derek Simpson (Amicus), Tony Woodley (TGWU) et Matt Wrack (FBU). 
politiques, et c'est d'ailleurs la position réformiste qui a toujours constitué la ligne majoritaire, notamment au sein des centrales syndicales les plus puissantes. La TULO, l'organisation qui assure le lien entre le parti et les syndicats, a pris ainsi depuis 2002 un rôle croissant dans la formulation des politiques travaillistes, et joue aujourd'hui le rôle de véritable groupe de pression syndical au sein du Parti travailliste.

\section{Un nouveau rapport de forces ?}

Les difficultés financières éprouvées depuis 2007 par le Parti travailliste peuvent sembler esquisser la possibilité d'un rééquilibrage des rapports entre les syndicats et le parti. Nombre des riches mécènes sur lesquels s'appuyait le Parti travailliste pour contrebalancer le poids financier des syndicats l'ont déserté suite à sa chute dans les sondages d'opinion. Les syndicats, qui sont intervenus pour sauver le Parti travailliste de la faillite, disposent donc de moyens de pression supplémentaires sur la direction travailliste. Au cours de l'été 2008, marqué par les conflits autour des salaires dans les services publics, les dirigeants des principaux syndicats affiliés au Parti travailliste - Unite et GMB notamment - ont demandé de nouveau une remise en cause des lois anti-syndicales mises en place dans les années 1980, et une inflexion des politiques menées par le gouvernement dans une direction plus progressiste.

Les menaces de rupture ou de retrait du soutien financier au Parti travailliste font cependant partie d'un jeu convenu entre les directions syndicales et le gouvernement travailliste. En dépit des craintes des éditorialistes conservateurs, les syndicats ne sont tout simplement pas aujourd'hui en mesure de dicter une politique sociale et économique au gouvernement travailliste. Les effectifs syndicaux se sont certes stabilisés depuis l'arrivée au pouvoir des travaillistes en 1997, mais si l'hémorragie s'est arrêtée, aucun regain militant n'est clairement identifiable. Alors que la Grande-Bretagne conserve une des législations les plus restrictives en Europe en matière de droits syndicaux, et que la protection conférée aux salariés leur provient essentiellement de droits individuels inscrits dans la loi, l'adhésion à un syndicat n'apparaît pas comme un impératif économique aux salariés britanniques. En l'absence d'une perspective de transformation radicale du cadre légal et économique de l'activité syndicale en Grande-Bretagne, les syndicats restent cantonnés au rôle d'acteurs marginaux sur la scène sociale britannique, et de partenaires subalternes dans leurs relations avec le gouvernement travailliste.

\section{Bibliographie}

BACHELOT, Carole. «Parti socialiste français et Parti travailliste britannique. Le cas des groupes dirigeants », Vingtième Siècle. Revue d'Histoire, ${ }^{\circ} 96$, octobredécembre 2007, pp. 107-121.

BRONFENBRENNER, K., FRIEDMAN, F., HURD, R., OSWALD, R. \& SEEBER R. (eds.). Organizing to Win: New Research on Union Strategies. Ithaca: New York, $1998,370 \mathrm{p}$. 
BUCKLEY, Christine. 'Big unions being urged by activists to split from the party', The Times, 19 mai 2008.

CLARK, L. \& DRURY, I. 'Here comes the summer of our discontent : As teachers' strike cripples our schools, Brown is warned of further public sector walkouts', Daily Mail, 25 avril 2008.

COATES, Sam. 'Unions call for Business Secretary John Hutton's head after "fallout", The Times, 23 juillet 2008.

CROUCH, Colin. The Politics of Industrial Relations. London: Fontana, 1979, $224 \mathrm{p}$.

FAUCHER-KING, Florence. «La 'modernisation' du Parti travailliste, 1997-2007. Succès et difficultés de l'importation du modèle entrepreneurial dans un parti politique », Politix, vol. 21, n ${ }^{\circ} 1,2008$, pp. 125-149.

FLOUD, R. \& JOHNSON, P. (ed). The Cambridge Economic History of Modern Britain. Volume III : Structural Change and Growth, 1939-2000. Cambridge : Cambridge University Press, 2004, xx, 476 p.

GOULD, Philip. The Unfinished Revolution: How the Modernisers Saved the Labour Party. London: Abacus 1999

GRICE, Andrew. 'Brown to reject calls for tax rise for higher paid', Independent, 25 juillet 2008.

GRICE, A., RUSSELL, B. \& MCSMITH, A. 'As strike bites, unions press Brown to raise taxes on rich', Independent, 17 juillet 2008.

HAY, Colin. "Narrating Crisis : The Discursive Construction of the "Winter of Discontent"', Sociology, vol. 30, no. 2, May 1996, pp. 253-277.

HEERY, Edmund. 'The Relaunch of the Trades Union Congress', British Journal of Industrial Relations, Vol. 36, No. 3, 1998, pp. 339-360.

HEERY, E., SIMMS, M. \& SIMPSON, D. Research Bulletin : New Unionism Research Project, No. 5, June 1999, Cardiff University.

HENCKE, David. 'MI5 opens door for union to represent spies', Guardian, 12 September 2008.

-. 'Political party funding : union cuts funds to MPs it says do not support Labour values', Guardian, 10 juin 2008.

—. 'Talks on future of public services turns fractious', Guardian, 26 juillet 2008.

HOWELL, Chris. Trade Unions and the State: The Construction of Industrial Relations Institutions in Britain, 1890-2000. Princeton: Princeton University Press, 2005, xii, 244 p.

JONES, Alan. 'GMB threatens Labour MPs' funds', The Independent, 10 juin 2008.

KAHN, P., LEWIS N., LIVOCK, R. \& WILES, P. avec l'assistance de MESHER J. Picketing. Industrial Disputes, Tactics and the Law, London: Routledge and Keegan Paul, 1983, xvi, 224 p. 
KELLY J. \& ANGELO B. 'A Long Summer of Discontent', Time, 30 juillet 1984.

KIRKUP, James. 'Labour "too dependent on trade unions", Daily Telegraph, 30 juin 2008.

-. 'Trade unions demand strike action rights in return for Labour Party donations', Daily Telegraph, 3 juillet 2008.

KISIEL, Ryan. 'Ministers bow to union leaders over new rights for workers', Daily Mail, 15 juillet 2008.

Labour Party. 1979 Labour Party Manifesto. London : Labour Party, 1979.

—. New Labour : Because Britain Deserves Better. London : Labour Party, 1997.

MANDELSON Peter. The Blair Revolution Revisited. Westminster: Politico's Publishing, 2002, 1, $268 \mathrm{p}$.

MORGAN, Kenneth O. Britain Since 1945 : The People's Peace. Oxford : Oxford University Press, 2001, 628 p.

National Union of Public Employees Archive, Modern Records Centre, University of Warwick, MSS.281/11.

OLIVER, Jonathan. 'Trade union chiefs ready to dump Brown', The Times, 20 juillet 2008.

PASCOE-WATSON, George. 'Brown snubs union strike call', Sun, 7 juillet 2008.

PIERCE, A. \& TYLER, R. 'Trade unions cash support for Labour in return for policy changes', Daily Telegraph, 13 juillet 2008.

PRINCE, Rosa. 'Unison union leaders threaten to withdraw Labour funding', Daily Telegraph, 10 juillet 2008.

REID, Alastair J. United We Stand: A History of Britain's Trade Unions. London: Penguin Books, 2005, xviii, 476 p.

STEWART, Heather. 'Dirty business: cleaning up the Square Mile's mess', Observer, 17 décembre 2006.

TAYLOR, Richard. The Trade Union Question in British Politics. Oxford : Blackwell, 1993, x, 406 p.

WADDINGTON J. \& HOFFMANN R. (ed). Trade Unions in Europe: facing challenges and searching for solutions. Bruxelles : European Trade Union Institute, 2000, 713 p.

WINTOUR, Patrick. 'Unions hit Brown with 130 demands', Guardian, 18 juillet 2008.

WINTOUR, P. \& WATT, N. 'Unions put new demands to Brown', Guardian, 30 juin 2008 . 